EPJ Web of Conferences 60, 18006 (2013)

DOI: $10.1051 /$ epjconf $/ 20136018006$

(C) Owned by the authors, published by EDP Sciences, 2013

\title{
Searches for direct pair production of third generation squarks with the ATLAS detector
}

\author{
Claudia Giuliani ${ }^{1, a}$ on behalf of the ATLAS Collaboration \\ ${ }^{1}$ Albert-Ludwigs-Universität Freiburg (DE)
}

\begin{abstract}
The most recent results of the searches for direct production of third generation squarks with the ATLAS detector are presented. All the selected analyses use $21 \mathrm{fb}^{-1}$ of pp collisions at $\sqrt{s}=8 \mathrm{TeV}$ and follow the assumptions of a Minimal Supersymmetric extension of the Standard Model of Particle Physics where the R-parity is conserved and the lightest supersymmetric particle (LSP) is the lightest neutralino. Naturalness considerations require that the partners of the top and bottom quarks are light $(O(1 \mathrm{TeV}))$ and hence have a relatively high production cross-section at the LHC. Since no deviations from the Standard Model predictions are observed, limits are set for different mass hierarchies and mass relations between the bottom and top squarks and the lightest chargino and neutralino.
\end{abstract}

\section{Introduction}

The results of the searches for direct stop and direct sbottom production with the ATLAS detector [1], all updated to the full $2012 \sqrt{s}=8 \mathrm{TeV}$ dataset of $\sim 20 \mathrm{fb}^{-1}$, are presented.

The searches are based on a Minimal Supersymmetric (SUSY) extension of the Standard Model (SM) of particle physics, where the R-parity is conserved. SUSY particles are therefore pair-produced and the lightest supersymmetric particle (LSP) is a stable, neutral and only weakly interacting particle and hence a good Dark Matter candidate.

Naturalness requires low fine tuning in the theory and implies a light higgsino sector, gluinos with a mass below $2 \mathrm{TeV}$ and light partners of the $3^{\text {rd }}$ generation SM quarks, making their production cross-section accessible for searches at the LHC.

Since no significant excess over the SM expectations was observed in any of the analyses, they were interpreted in different scenarios and exclusion limits were set. For direct sbottom $\left(\tilde{b}_{1}\right)$ production (Sect. 2 ), the decays considered are $\tilde{b}_{1} \rightarrow b+\tilde{\chi}_{1}^{0}$ [2], where $\tilde{\chi}_{1}^{0}$ is the lightest neutralino, and $\tilde{b}_{1} \rightarrow t+\tilde{\chi}_{1}^{ \pm}, \tilde{\chi}_{1}^{ \pm} \rightarrow W^{(*)}+\tilde{\chi}_{1}^{0}$ [3], where $\tilde{\chi}_{1}^{ \pm}$is the lightest chargino.

The direct stop $\left(\tilde{t}_{1}\right)$ searches (Sect. 3) target mainly two scenarios: $\tilde{t}_{1} \rightarrow t+\tilde{\chi}_{1}^{0}[4,5]$ and $\tilde{t}_{1} \rightarrow b+\tilde{\chi}_{1}^{ \pm}, \tilde{\chi}_{1}^{ \pm} \rightarrow$ $W^{(*)}+\tilde{\chi}_{1}^{0}[2,5,6]$. Different $\tilde{t}_{1}-\tilde{\chi}_{1}^{ \pm}-\tilde{\chi}_{1}^{0}$ mass relations are considered. When the mass difference between the stop and the neutralino is smaller than the mass of the top quark, the decay $\tilde{t}_{1} \rightarrow t+\tilde{\chi}_{1}^{0}$ through an on-shell top is suppressed, whilst the three body decay (through an off-shell top) $\tilde{t}_{1} \rightarrow b+W+\tilde{\chi}_{1}^{0}$ opens up. Results for the interpretation in a three-body decay scenario have been presented

\footnotetext{
ae-mail: claudia.giuliani@ cern.ch
}

for the first time at LHCP 2013 [6]. The chosen results include also interpretations in more complex direct stop production scenarios for which $Z$-bosons are present in the decay chain [7], e.g. $\tilde{t}_{2} \rightarrow \tilde{t}_{1} Z, \tilde{t}_{1} \rightarrow t \tilde{\chi}_{1}^{0}$, powerful in covering the region $m\left(\tilde{t}_{1}\right)-m\left(\tilde{\chi}_{1}^{0}\right)=m(t)$, and Natural GMSB scenarios $\tilde{t}_{1} \rightarrow X Z \tilde{G}$, where $\tilde{G}$ is the gravitino.

\section{Searches for direct sbottom production}

The direct sbottom production and decay scenarios have been covered by two analyses, one focusing on final states with two leptons with the same charge and jets (Sect. 2.1) and one that requires two b-tagged jets and high $E_{\mathrm{T}}^{\text {miss }}$ (Sect. 2.2).

\subsection{Searches in final states with 2 same-sign leptons and jets}

The search for Supersymmetry in final states with two Same Sign (SS) leptons [3] has interpretations in many SUSY production and decay scenarios. Here only the interpretation for direct sbottom production with $\tilde{b}_{1} \rightarrow$ $t+\tilde{\chi}_{1}^{ \pm}, \tilde{\chi}_{1}^{ \pm} \rightarrow W^{(*)}+\tilde{\chi}_{1}^{0}$, with branching ratio $\mathrm{BR}=1$, is considered.

This analysis exploits the low SM background to get a good sensitivity to SUSY processes. The only sources of SS leptons in the Standard Model are the associated production of $t \bar{t}+$ vector-boson and the diboson production, that have to be added to the contributions containing misidentified leptons. These consist of processes where the SS pair arises either from charge-misidentification (radiation of a hard photon bremsstrahlung followed by an asymmetric conversion) or from a non-prompt lepton mainly produced in a $b$-hadron decay. 

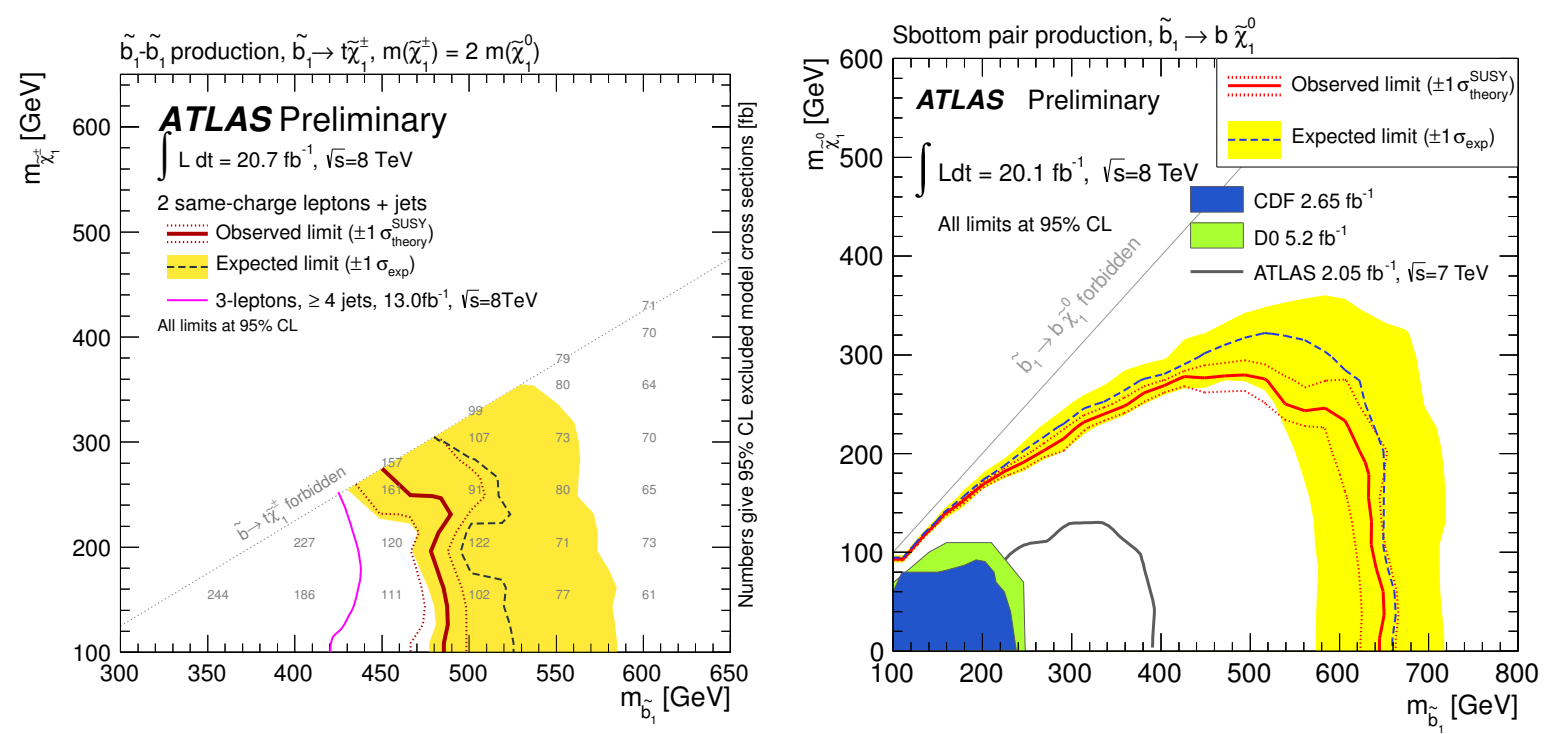

Figure 1. Expected (dashed lines) and observed (solid lines) limits at 95\% CL for direct sbottom pair production. On the left the limit for the $\tilde{b}_{1} \rightarrow t \tilde{\chi}_{1}^{ \pm}$model with $m\left(\tilde{\chi}_{1}^{ \pm}\right)=2 \cdot m\left(\tilde{\chi}_{1}^{0}\right)$ is shown [3]; the numbers give the limits on the excluded model cross sections in fb. The right plot shows the limit obtained in the $\tilde{b}_{1}-\tilde{\chi}_{1}^{0}$ plane assuming $\tilde{b}_{1} \rightarrow b \tilde{\chi}_{1}^{0}$ with $100 \% \mathrm{BR}$ [2].

The signal selection requires, on top of the SS lepton pair, the presence of at least three jets with $p_{\mathrm{T}}>40 \mathrm{GeV}$, of which one identified as b-jet with $p_{\mathrm{T}}>20 \mathrm{GeV}$, $E_{\mathrm{T}}^{\mathrm{miss}}>150 \mathrm{GeV}$,

$m_{\mathrm{T}}\left(\ell_{1}, E_{\mathrm{T}}^{\text {miss }}\right)=\sqrt{2 p_{\mathrm{T}}^{\ell} E_{\mathrm{T}}^{\text {miss }}-2 \mathbf{p}_{\mathrm{T}}^{\ell} \cdot \mathbf{p}_{\mathrm{T}}^{\text {miss }}}>100 \mathrm{GeV}$ and high effective mass, $m_{e f f}=\sum_{i=1}^{2} p_{\mathrm{T}}^{\ell_{i}}+\sum_{k=1}^{n-j e t s} p_{\mathrm{T}}^{\text {jet }}+E_{\mathrm{T}}^{\mathrm{miss}}$, in the event.

To maximise the exclusion reach, a shape fit in $m_{e f f}$ is performed. The limits are computed as $95 \%$ Confidence Levels (CL), using the $C L_{s}$ method, with a fit based on the profile likelihood ratio [13]. Exclusion limits are extracted for two different signal assumptions in the $m\left(\tilde{b}_{1}\right)-m\left(\tilde{\chi}_{1}^{ \pm}\right)$ plane. For a fixed neutralino mass $m\left(\tilde{\chi}_{1}^{0}\right)=60 \mathrm{GeV}$ sbottom masses up to $470 \mathrm{GeV}$ are excluded at $95 \% \mathrm{CL}$ for all the allowed chargino masses. Figure 1 left shows the excluded area for the model $m\left(\tilde{\chi}_{1}^{ \pm}\right)=2 \cdot m\left(\tilde{\chi}_{1}^{0}\right)$ : sbottom masses up to $450 \mathrm{GeV}$ are excluded at $95 \% \mathrm{CL}$ for the allowed $\tilde{\chi}_{1}^{ \pm}$masses.

\subsection{Searches in final states with 2 b-jets and $E_{\mathrm{T}}^{\text {miss }}$}

The search focusing on final states with 2 b-tagged jets, $E_{\mathrm{T}}^{\mathrm{miss}}$ and very low additional hadronic activity [2] is developed to investigate direct sbottom and direct stop production, for which the results are reported in section 3.1.

The scenario considered for direct sbottom production and decay is $\tilde{b}_{1} \rightarrow b \tilde{\chi}_{1}^{0}, \mathrm{BR}=1$, and limits are extracted in the $m\left(\tilde{b}_{1}\right)-m\left(\tilde{\chi}_{1}^{0}\right)$ plane.

The main backgrounds to this analysis come from the semi-leptonic decay of $t \bar{t}$ pairs, where the lepton is either an hadronic decaying $\tau$ or is lost or misidentified, and $Z \rightarrow v v$ and $W$ production in association with b-jets.

Two different signal selections (SR) are defined, one sensitive to large mass splitting between the relevant SUSY particles, in this case $\tilde{b}_{1}$ and $\tilde{\chi}_{1}^{0}$, and one that exploits an initial state radiation (ISR) signature, that is, a hard jet emitted back to back to the $\tilde{b}_{1} \tilde{b}_{1}^{*}$ system giving higher $E_{\mathrm{T}}^{\text {miss }}$ in the event, and is sensitive to compressed scenarios. For both SRs the selected events must pass the requirements for a $E_{\mathrm{T}}^{\text {miss }}$ trigger, not contain any lepton, have two b-tagged jets, and have almost no additional hadronic activity on top of the requested jets. The selection for large mass splittings scenarios uses the contransverse mass [14] of the two b-jets, $m_{\mathrm{CT}}^{2}\left(v_{1}, v_{2}\right)=$ $\left[E_{\mathrm{T}}\left(v_{1}\right)+E_{\mathrm{T}}\left(v_{2}\right)\right]^{2}-\left[\mathbf{p}_{\mathrm{T}}\left(v_{1}\right)-\mathbf{p}_{\mathrm{T}}\left(v_{2}\right)\right]^{2}$, as main discriminating variable against $t \bar{t}$, and the invariant mass of the $b b$ system, $m_{b b}>200 \mathrm{GeV}$, against the $\mathrm{Z}$ background. Five signal enhanced selections are defined in step of 50 $\mathrm{GeV}$ for increasing values of $m_{\mathrm{CT}}$ starting from $m_{\mathrm{CT}}>$ $150 \mathrm{GeV}$. For the ISR selection, the leading jet is required to be a high $p_{\mathrm{T}}$ non b-tagged jet with a big angular separation with the $E_{\mathrm{T}}^{\mathrm{miss}}$, and the b-tagged jets are selected as the second and third leading jets. A tight requirement is then applied on $E_{\mathrm{T}}^{\mathrm{miss}}>250 \mathrm{GeV}$ and on the scalar sum of the momenta carried by the additional jets in the event, $H_{\mathrm{T}, 3}=\sum_{i=4}^{n-\text { jets }}\left(p_{\mathrm{T}}^{\text {jet }}\right)_{i}<50 \mathrm{GeV}$.

No significant excess over the SM model was observed and $95 \%$ CL limits were set using the $C L_{s}$ prescription. The obtained exclusion curve is shown in figure 1 on the right. Sbottom masses up to $620 \mathrm{GeV}$ are excluded for neutralino masses up to $220 \mathrm{GeV}$.

\section{Searches for direct stop production}

Searches for direct stop production are performed for final states with 0,1,2 and 3 leptons. The area of the parameter space excluded by the stop searches at 95\% CL is summarised in figure 2 for the $\tilde{t}_{1} \rightarrow t \tilde{\chi}_{1}^{0}$ and $\tilde{t}_{1} \rightarrow b \tilde{\chi}_{1}^{ \pm}$models and in figure 3 for the searches in final states with a $Z$ boson. 


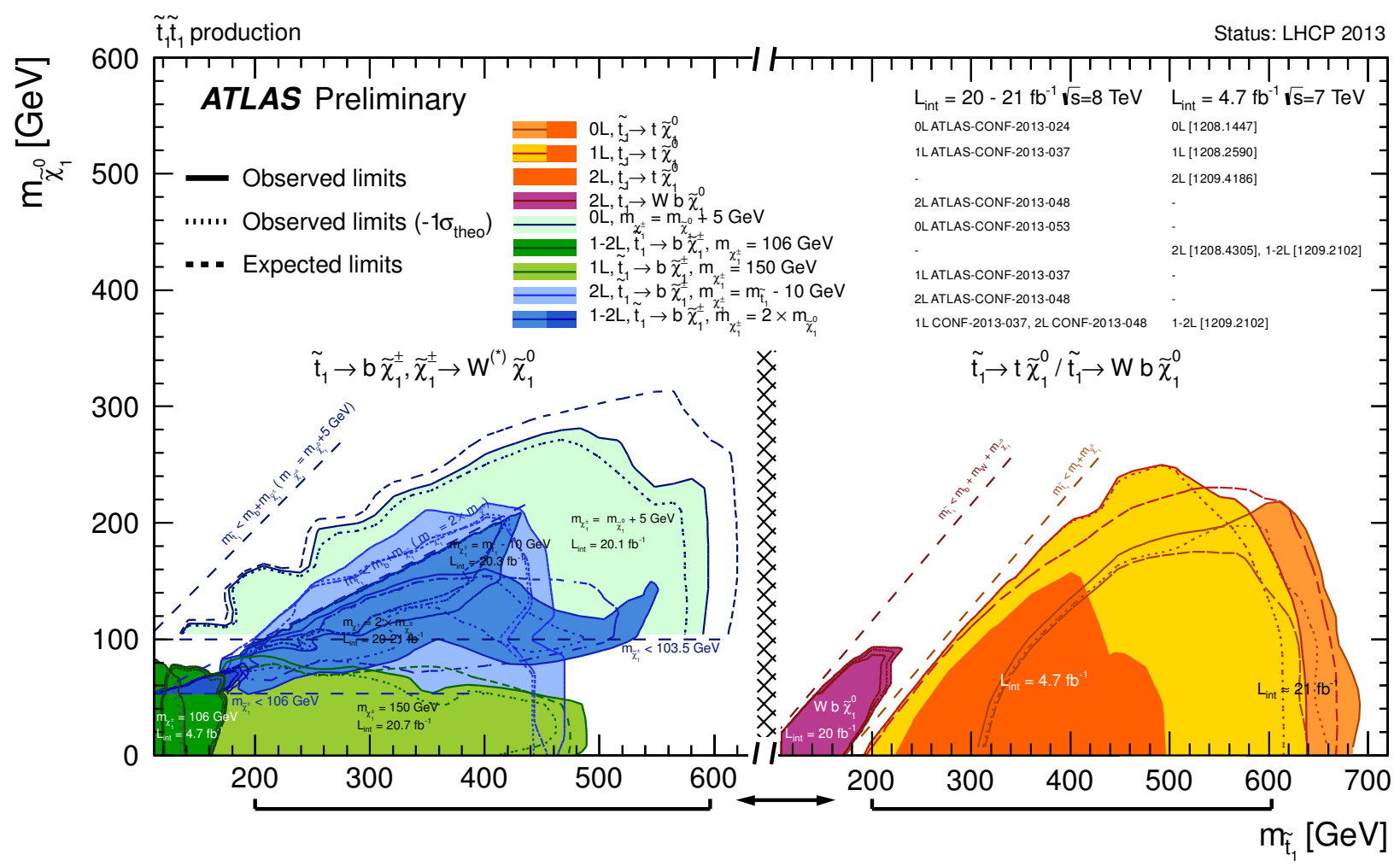

Figure 2. Summary of the dedicated ATLAS searches for top squark (stop) pair production based on up to $13-21 \mathrm{fb}^{-1}$ of $\mathrm{pp}$ collision data taken at $\sqrt{s}=8 \mathrm{TeV}$ and $4.7 \mathrm{fb}^{-1}$ of pp collision data taken at $\sqrt{s}=7 \mathrm{TeV}$. Exclusion limits at 95\% CL are shown in the $\tilde{t}_{1}-\tilde{\chi}_{1}^{0}$ mass plane. The dashed and solid lines show the expected and observed limits, respectively, including all uncertainties except the theoretical signal cross section uncertainty (PDF and scale). The dotted lines represent the results obtained when reducing the nominal signal cross section by $1 \sigma$ of its theoretical uncertainty. Three decay modes are considered separately with $100 \%$ BR: $\tilde{t}_{1} \rightarrow t+\tilde{\chi}_{1}^{0}$ (7 TeV: [8-10], $8 \mathrm{TeV}[4,5]$, where the $\tilde{t}_{1}$ is mostly $\left.\tilde{t}_{R}\right), \tilde{t}_{1} \rightarrow W+b+\tilde{\chi}_{1}^{0}$ (3-body decay for $\left.m\left(\tilde{t}_{1}\right)<m(t)+m\left(\tilde{\chi}_{1}^{0}\right), 8 \mathrm{TeV}[6]\right)$, and $\tilde{t}_{1} \rightarrow b+\tilde{\chi}_{1}^{ \pm}, \tilde{\chi}_{1}^{ \pm} \rightarrow W^{(*)}+\tilde{\chi}_{1}^{0}$. In the latter case, several hypotheses on the $\tilde{t}_{1}, \tilde{\chi}_{1}^{ \pm}, \tilde{\chi}_{1}^{0}$ mass hierarchy are assumed: fixed $\tilde{\chi}_{1}^{ \pm}$mass $(106$ $\mathrm{GeV}[11,12], 150 \mathrm{GeV}[5]), m\left(\tilde{\chi}_{1}^{ \pm}\right) \sim 2 \times m\left(\tilde{\chi}_{1}^{0}\right)[5,6,12]$, fixed $m\left(\tilde{t}_{1}\right)-m\left(\tilde{\chi}_{1}^{ \pm}\right)=10 \mathrm{GeV}$ [6], and fixed $m\left(\tilde{\chi}_{1}^{ \pm}\right)-m\left(\tilde{\chi}_{1}^{0}\right)=5 \mathrm{GeV}[2]$.

\subsection{Searches in final states with 2 b-jets and $E_{\mathrm{T}}^{\text {miss }}$}

The structure of the $2 \mathrm{~b}$-jets and $E_{\mathrm{T}}^{\mathrm{miss}}$ analysis has already been described in detail in section 2.2 in relationship to the interpretation in a direct sbottom production model. This final state is powerful also to detect direct stop production in the scenarios $\tilde{t}_{1} \rightarrow b \tilde{\chi}_{1}^{ \pm}, \tilde{\chi}_{1}^{ \pm} \rightarrow W^{(*)} \tilde{\chi}_{1}^{0}$, with $\mathrm{BR}=1$, where the mass difference between the chargino and the neutralino becomes small and therefore the decay products of the $W$ get lost [2].

The exclusion contour at $95 \% \mathrm{CL}$ in the $\tilde{t}_{1}-\tilde{\chi}_{1}^{0}$ mass plane for a fixed $m\left(\tilde{\chi}_{1}^{ \pm}\right)-m\left(\tilde{\chi}_{1}^{0}\right)=5 \mathrm{GeV}$ covers stop masses up to $580 \mathrm{GeV}$ and neutralino masses up to $250 \mathrm{GeV}$ for a mass of the stop of $500 \mathrm{GeV}$, see figure 2 teal-coloured contour. When considering a $m\left(\tilde{\chi}_{1}^{ \pm}\right)-m\left(\tilde{\chi}_{1}^{0}\right)=20 \mathrm{GeV}$ the excluded area shrinks and stop masses up to $420 \mathrm{GeV}$ are excluded at $95 \%$ CL for neutralino masses below 200 $\mathrm{GeV}$. The simulated signal grids respect the LEP limit on the mass of the $\tilde{\chi}_{1}^{ \pm}$just above $100 \mathrm{GeV}$.

\subsection{Searches in final states with jets and $E_{\mathrm{T}}^{\text {miss }}$}

The search for direct stop production in all hadronic final states with high missing transverse momentum [4] has an interpretation for $\tilde{t}_{1} \rightarrow t \tilde{\chi}_{1}^{0}$ with $\mathrm{BR}=1$, orange curve in figure 2 .

The main background sources to the analysis come from the $t \bar{t}$ semi-leptonic decay, the associated production of $Z \rightarrow v v$ with b-jets, the associated production of $t \bar{t}+$ vectorboson, which is the only irreducible background, and fake sources such as multi-jet production and all-hadronic $t \bar{t}$. The signal signature is obtained requiring high $E_{\mathrm{T}}^{\mathrm{miss}}$ and six jets of which at least two b-tagged, the two leading ones with a $80 \mathrm{GeV}$ transverse momentum threshold, the others with $p_{\mathrm{T}}>35 \mathrm{GeV}$. The rejection of the semileptonic decaying top and the electroweak background is achieved by vetoing the events with a $\tau$ lepton, by requiring that the transverse mass between the nearest $b$ jet to the $E_{\mathrm{T}}^{\text {miss }}$ and the $E_{\mathrm{T}}^{\text {miss }}$ itself is above the top mass $\left(m_{\mathrm{T}}\left(b_{\text {near }}, E_{\mathrm{T}}^{\mathrm{miss}}\right)>175 \mathrm{GeV}\right)$ and by reconstructing the two hadronic decaying tops. The final discriminating variable is $E_{\mathrm{T}}^{\mathrm{miss}}$ and inclusive signal regions are defined for increasing values of the missing transverse momentum, $E_{\mathrm{T}}^{\text {miss }}>200,300,350 \mathrm{GeV}$.

Exclusion limits at $95 \% \mathrm{CL}$ were placed on the $m\left(\tilde{t}_{1}\right)-$ $m\left(\tilde{\chi}_{1}^{0}\right)$ plane for stop masses below $670 \mathrm{GeV}$ for $m\left(\tilde{\chi}_{1}^{0}\right)=$ $1 \mathrm{GeV}$ and for neutralino masses up to $200 \mathrm{GeV}$ for a mass of the stop of $600 \mathrm{GeV}$, see orange contour of figure 2. The generated signal grid has a stop decaying mostly in a top 
with right polarisation. It has been verified that the analysis results are independent on the top polarisation choice. A study has then been done to determine the maximum branching ratio excluded at $95 \% \mathrm{CL}$ assuming that the analysis is completely insensitive to the decay products not coming from the $\tilde{t}_{1} \rightarrow t \tilde{\chi}_{1}^{0}$ decay. For a BR of $75 \%$ stop masses between $340 \mathrm{GeV}$ and $600 \mathrm{GeV}$ are excluded for an almost massless neutralino.

\subsection{Searches in final states with 1 lepton, jets and $E_{\mathrm{T}}^{\text {miss }}$}

The search for direct stop production in final states with one lepton, jets and $E_{\mathrm{T}}^{\mathrm{miss}}$ [5] is the analysis able to cover better the bulk of the $\tilde{t}_{1}, \tilde{\chi}_{1}^{ \pm}, \tilde{\chi}_{1}^{0}$ parameter space. It has interpretations for $\tilde{t}_{1} \rightarrow t \tilde{\chi}_{1}^{0}$ and for $\tilde{t}_{1} \rightarrow b \tilde{\chi}_{1}^{ \pm}$scenarios. The baseline signal selection requires exactly one well identified and isolated lepton, $e$ or $\mu$, with $p_{\mathrm{T}}>25 \mathrm{GeV}, 4$ jets or more, the four leading with $p_{\mathrm{T}}>80,60,40,25 \mathrm{GeV}$; among them at least one or two, depending on the signal model under consideration, has to be identified as bjet. The main background processes to the analysis arise, therefore, from $t \bar{t}$ and $W+$ jets production.

To suppress the SM contributions, a series of selections are further applied. The most powerful variables used to reject the $t \bar{t}$ and $W$ backgrounds are the $E_{\mathrm{T}}^{\text {miss }}$ and the transverse mass between the lepton and the $E_{\mathrm{T}}^{\mathrm{miss}}$. The analysis makes also use of a $\tau$ veto and of two asymmetric version of the $m_{\mathrm{T} 2}$ variable $[15,16]$. The $m_{\mathrm{T} 2}$ variable is powerful to discriminate against the semi-leptonic $t \bar{t}$ background since the variable presents a sharp endpoint for the top and not for the signal. The endpoint depends on the mass of the invisible particles in the $t \bar{t}$ decay, while for the signal the dependancy comes from the $\tilde{t}_{1}-\tilde{\chi}_{1}^{0}$ or $\tilde{\chi}_{1}^{ \pm}-\tilde{\chi}_{1}^{0}$ mass difference. It is defined as

$$
m_{\mathrm{T} 2} \equiv \min _{\vec{p}_{T, a}^{C}+\vec{p}_{T, b}^{C}=\vec{p}_{\mathrm{T}}^{\text {miss }}}\left\{\max \left(m_{T, a}, m_{T, b}\right)\right\}
$$

and the two variations considered are $a m_{\mathrm{T} 2}$ and $m_{\mathrm{T} 2}^{\tau}$. The former is an asymmetric definition where the missing particles in the dileptonic top decay are defined as the $W$ and the neutrinos and has the end point at $m(t)$; the latter is defined for dileptonic top decays where one of the leptons is an hadronic decaying $\tau$ and in this case the end point of the top distribution is at $m(W)$.

Different thresholds and combinations of the discriminating variables are chosen depending on the targeted scenario $\tilde{t}_{1} \rightarrow t \tilde{\chi}_{1}^{0}$ or $\tilde{t}_{1} \rightarrow b \tilde{\chi}_{1}^{ \pm}$and three signal enhanced regions are defined for each of the two signal models. For $\tilde{t}_{1} \rightarrow t \tilde{\chi}_{1}^{0}$ a SR is defined in bins of $m_{\mathrm{T}}$ and $E_{\mathrm{T}}^{\text {miss }}$ and a combined shape fit is performed to extend the analysis sensitivity as much as possible towards the region $m\left(\tilde{t}_{1}\right) \sim m(t)+m\left(\tilde{\chi}_{1}^{0}\right)$.

In the $\tilde{t}_{1} \rightarrow t \tilde{\chi}_{1}^{0}$ scenario, for an almost massless neutralino, stop masses are excluded at 95\% CL between 200 and $620 \mathrm{GeV}$ and for a stop mass of $500 \mathrm{GeV}$ a neutralino is excluded up to $250 \mathrm{GeV}$, as shown in figure 2 with a dark yellow contour. As for the stop search in all hadronic final states, described in section 3.2, the signal grid has been simulated with a stop decaying mainly in $t_{R}$. This analysis is found to be sensitive to the top polarisation; when considering a stop decaying only in left polarised top, a loss in acceptance is observed that translates in a weaker upper limit on the stop mass of about $50 \mathrm{GeV}$.

The exclusion contour at $95 \% \mathrm{CL}$ for the $\tilde{t}_{1} \rightarrow b \tilde{\chi}_{1}^{ \pm}, \tilde{\chi}_{1}^{ \pm} \rightarrow$ $W^{(*)} \tilde{\chi}_{1}^{0}$ model considering $m\left(\tilde{\chi}_{1}^{ \pm}\right)=2 \cdot m\left(\tilde{\chi}_{1}^{0}\right)$, figure 2 blue contour, greatly extends the area excluded with the $7 \mathrm{TeV}$ ATLAS dataset, dark blue contour. Now stop masses between 200 and almost $500 \mathrm{GeV}$ are excluded at 95\% CL for neutralino masses ranging between 70 and $140 \mathrm{GeV}$. The light green contour of figure 2 shows the excluded area at $95 \% \mathrm{CL}$ for a fixed $m\left(\tilde{\chi}_{1}^{ \pm}\right)=150 \mathrm{GeV}$, here the analysis is able to exclude stop decaying in $b \tilde{\chi}_{1}^{ \pm}$up to a mass of $450 \mathrm{GeV}$ for a $40 \mathrm{GeV}$ neutralino.

\subsection{Searches in final states with 2 opposite charged leptons and $E_{\mathrm{T}}^{\text {miss }}$}

Final states with two opposite charged (OS) leptons allow to select a clean signature without the requirement of high jet multiplicity and $E_{\mathrm{T}}^{\mathrm{miss}}$ in the event. The stop search that exploits the two lepton selection [6] is therefore sensitive to all the scenarios were jets are soft or are out of the detector acceptance and hence not correctly reconstructed. In particular, a good sensitivity is reached for the $\tilde{t}_{1} \rightarrow b \tilde{\chi}_{1}^{ \pm}, \tilde{\chi}_{1}^{ \pm} \rightarrow W^{(*)} \tilde{\chi}_{1}^{0}$ model when assuming a small $\Delta m\left(\tilde{t}_{1}, \tilde{\chi}_{1}^{ \pm}\right)$and for the region $m\left(\tilde{t}_{1}\right) \sim m(b)+m\left(\tilde{\chi}_{1}^{ \pm}\right)$ with $m\left(\tilde{\chi}_{1}^{ \pm}\right)=2 \cdot m\left(\tilde{\chi}_{1}^{0}\right)$. For the first time a limit under the assumption of a three-body decay of the stop in $\tilde{t}_{1} \rightarrow b+W+\tilde{\chi}_{1}^{0}$ is extracted. The three-body decay channel opens when the mass difference between the stop and the neutralino becomes smaller than the mass of the top and the $\tilde{t}_{1} \rightarrow t \tilde{\chi}_{1}^{0}$ decay with an off-shell top is disfavoured with respect to the stop three-body decay in $b+W+\tilde{\chi}_{1}^{0}$. The main SM backgrounds in this channel are the dileptonic top and the diboson production. The signal selection is based on the requirement of the lepton pair and uses as discriminating variable

$m_{\mathrm{T} 2}\left(\mathbf{p}_{\mathrm{T}}^{\ell_{1}}, \mathbf{p}_{\mathrm{T}}^{\ell_{2}}, \mathbf{p}_{\mathrm{T}}^{\text {miss }}\right)=\min _{\mathbf{q}_{\mathrm{T}}+\mathbf{r}_{\mathrm{T}}=\mathbf{p}_{\mathrm{T}}^{\text {miss }}}\left\{\max \left[m_{\mathrm{T}}\left(\mathbf{p}_{\mathrm{T}}^{\ell_{1}}, \mathbf{q}_{\mathrm{T}}\right), m_{\mathrm{T}}\left(\mathbf{p}_{\mathrm{T}}^{\ell_{2}}, \mathbf{r}_{\mathrm{T}}\right)\right]\right\}$

that has an endpoint at the $\mathrm{W}$ mass for the $t \bar{t}$ background. Four signal enhanced region are defined by increasing the lower threshold of the $m_{\mathrm{T} 2}$ variable; the most inclusive SR is defined for $m_{\mathrm{T} 2}>90 \mathrm{GeV}$ and has no requirements on jets, the tighter SRs require $m_{\mathrm{T} 2}>100,110,120 \mathrm{GeV}$ and the presence of two jets in the event.

In the $\tilde{t}_{1} \rightarrow b \tilde{\chi}_{1}^{ \pm}$model for $m\left(\tilde{\chi}_{1}^{ \pm}\right)=2 \cdot m\left(\tilde{\chi}_{1}^{0}\right)$, the analysis excludes at $95 \% \mathrm{CL}$ on the diagonal $m\left(\tilde{t}_{1}\right)=m_{b}+m\left(\tilde{\chi}_{1}^{ \pm}\right)$ stop masses between 200 and $420 \mathrm{GeV}$, as shown in figure 2 blue contour; the light blue area of figure 2 represent, instead, the excluded region at $95 \% \mathrm{CL}$ under the constraint $m\left(\tilde{t}_{1}\right)-m\left(\tilde{\chi}_{1}^{ \pm}\right)=10 \mathrm{GeV}$ for which stop masses up to 440 $\mathrm{GeV}$ are discarded for an almost massless neutralino.

The purple contour of figure 2 is the $95 \%$ CL limit obtained for the three-body decay scenario where stop masses up to $200 \mathrm{GeV}$ are excluded for almost all allowed neutralino masses. 

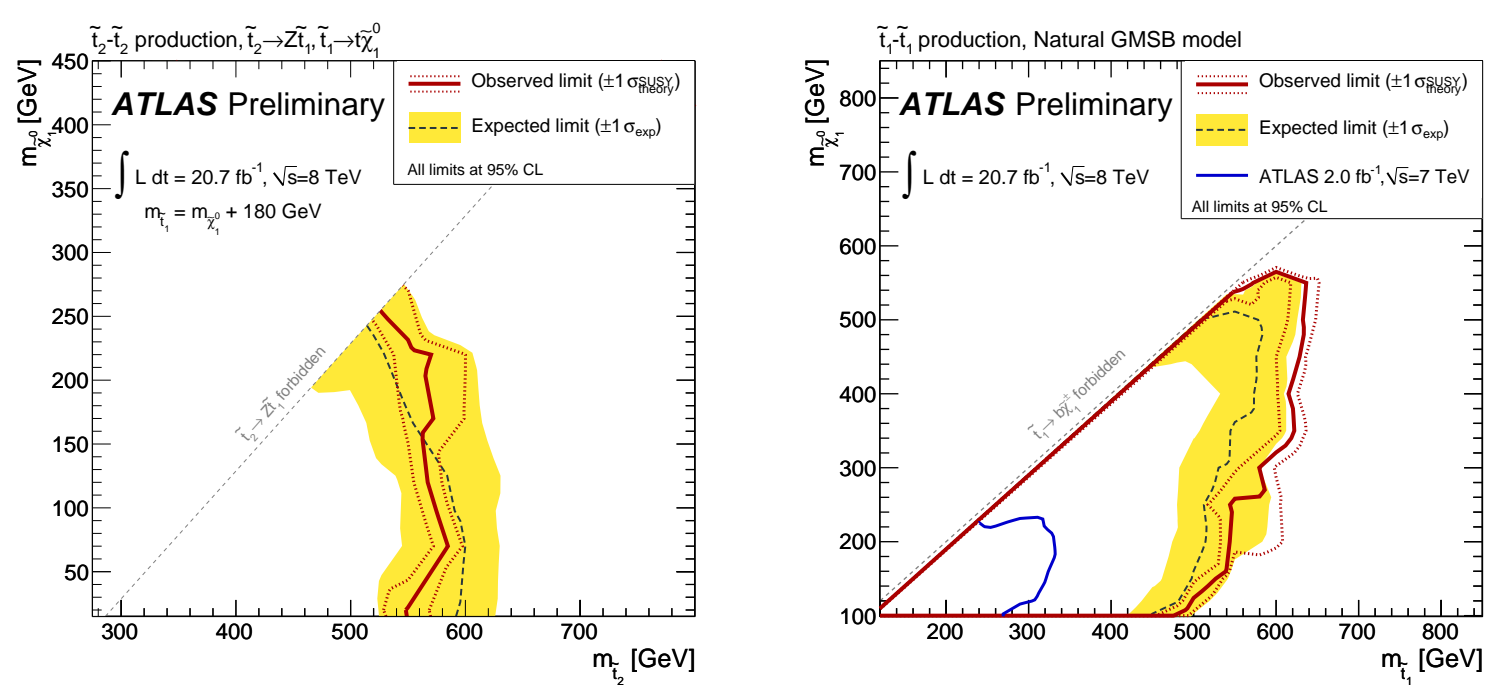

Figure 3. Expected (dashed lines) and observed (solid lines) limits at 95\% CL for the $m\left(\tilde{t}_{2}\right)-m\left(\tilde{\chi}_{1}^{0}\right)$ plane for the $\tilde{t}_{2}$ analysis, on the left, and for the stop natural GMSB model, on the right [7].

\subsection{Searches in decay chains with Z}

The search for direct stop production in decay chains with a $Z$-boson exploits final states where at least two leptons are present [7]. Two different models are considered, the direct pair production and decay of $\tilde{t}_{2} \rightarrow$ $Z \tilde{t}_{1}, \tilde{t}_{1} \rightarrow t \tilde{\chi}_{1}^{0}$, particularly interesting for covering the region $m\left(\tilde{t}_{1}\right)-m\left(\tilde{\chi}_{1}^{0}\right)=m(t)$, and a natural GMSB (GaugeMediated Supersymmetry Breaking) scenario where a pair of $\tilde{t}_{1}$ is produced and decays in final states with a $\mathrm{Z}$ and a gravitino, in this case the LSP.

The signal selection requires two oppositely charged leptons, whose invariant mass is compatible with the $Z$-boson mass, jets, of which at least one identified as b-jet, and high $E_{\mathrm{T}}^{\text {miss }}$; for the $\tilde{t}_{2}$ model an additional lepton is also required. The main SM backgrounds come from $t \bar{t}$, also in association with vector boson, and diboson production.

The obtained exclusion limits at $95 \%$ CL are show in figure 3 ; on the left, for $\tilde{t}_{2} \rightarrow Z \tilde{t}_{1}$, with $m\left(\tilde{t}_{1}\right)=m\left(\tilde{\chi}_{1}^{0}\right)+$ $180 \mathrm{GeV}, \tilde{t}_{2}$ masses are excluded up to $530 \mathrm{GeV}$ for all the allowed neutralino masses; in the natural GMSB scenario the extracted exclusion is for $\tilde{t}_{1}$ masses up to $450 \mathrm{GeV}$ for all the allowed neutralino masses, as shown in the right plot.

\section{References}

[1] ATLAS Collaboration, 2008 JINST 3 S08003

[2] ATLAS Collaboration, ATLAS-CONF-2013-053, http://cds.cern.ch/record/1547570
[3] ATLAS Collaboration, ATLAS-CONF-2013-007, https://cds.cern.ch/record/1522430

[4] ATLAS Collaboration, ATLAS-CONF-2013-024, https://cds.cern.ch/record/1525880

[5] ATLAS Collaboration, ATLAS-CONF-2013-037, https://cds.cern.ch/record/1532431

[6] ATLAS Collaboration, ATLAS-CONF-2013-048, http://cds.cern.ch/record/1547564

[7] ATLAS Collaboration, ATLAS-CONF-2013-025, https://cds.cern.ch/record/1525881

[8] ATLAS Collaboration, PRL 109, 211802 (2012), arXiv:1208.1447

[9] ATLAS Collaboration, PRL 109, 211803 (2012), arXiv:1208.2590

[10] ATLAS Collaboration, JHEP 11, 094 (2012), arXiv:1209.4186

[11] ATLAS Collaboration, EPJC 72, 2237 (2012), arXiv:1208.4305

[12] ATLAS Collaboration, PLB 720,13 (2013), arXiv:1209.2102

[13] G. Cowan, K. Cranmer, E. Gross and O. Vitells, Eur. Phys. J. C71, 1554 (2011), arXiv:1007.1727

[14] G. Polesello, D. R. Tovey, JHEP 03, 30 (2010), arXiv:0910.0174

[15] C. G. Lester, D. J. Summers, Phys.Lett.B 463, 99103 (1999), arXiv:hep-ph/9906349

[16] A. Barr, C. Lester, P. Stephens, J.Phys.G 29, 23432363 (2003), arXiv:hep-ph/0304226 